\title{
Investigating Older and Younger Peoples' Motivations for Lifelogging with Wearable Cameras
}

\author{
Niamh Caprani, Noel E. O’Connor, \& Cathal Gurrin \\ CLARITY: Centre for Sensor Web Technologies \\ Dublin City University \\ Ireland
}

\begin{abstract}
People have a natural tendency to collect things about themselves, their experiences and their shared experiences with people important to them, especially family. Similar to traditional objects such as photographs, lifelogs have been shown to support reminiscence. A lifelog is a digital archive of a person's experiences and activities and lifelog devices such as wearable cameras can automatically and continuously record events throughout a whole day. We were interested in investigating what would motivate people to lifelog. Due to the importance of shared family reminiscence between family members we focused our study on comparing shared or personal motivations with ten older and ten younger family members. We found from our results that both older and younger adults were more likely to lifelog for the purposes of information sharing and that reviewing lifelog images supported family reminiscence, reflection and story-telling. Based on these findings, recommendations are made for the design of a novel intergenerational family lifelog system.
\end{abstract}

Keywords-wearable cameras; image sharing; reminiscence

\section{INTRODUCTION}

With the population ageing across the developing world it is expected that by the 2060s we will experience a grandparent boom. By 2060 it is projected that there will be two or more elderly adults to every child compared to an average of three youngsters to every elderly adult in the 1960s [1]. This shift will no doubt change the structure of the family unit. It will perhaps become common for a 45-year-old individual to help their 70-year-old parent who conversely cares for the 95-yearold grandparent. In relation to lifelogging research, this change in family structure provides an opportunity to explore intergenerational family lifelogging and sharing. Lifelogging is the automatic capture of life experiences through devices and sensors either worn on the body or in the environment. In contrast to surveillance technologies, lifelogging devices capture information for personal use from a personal perspective. A smart phone for example can record your location (GPS), your movements (accelerometer), as well as your photographs, videos, text messages, emails and todo-lists to name but a few. As smart phones are currently ubiquitous, several projects are underway to increase the battery life of these devices to support lifelogging, allowing automatic capture of events throughout a whole day [2, 3]. Automated, wearable cameras can be used to capture a visual record of a person's day from a first-person point of view without any user intervention. The advantage of wearable cameras over other devices investigated for the purpose of visual lifelogging, such as mobile phones, is the longevity of battery life. They also hold advantages over video recorders, as they only take images on average twice per minute, which means that storage requirements are reduced, and privacy concerns are not as great, as the camera takes snapshots as opposed to continuous footage. Automated, wearable cameras are now used not only by lifelogging research groups, but also by research groups in other fields, as they presently offer the most usable and accessible visual lifelog capture solution. The limited functions of these devices also make them ideal for people who have limited or no technology experience.

Although lifelog information may not initially provide meaning for the person collecting it, over time patterns begin to develop and after many years these lifelogs could provide a rich narrative of a person's life. One of the potential benefits of lifelog data is to support reminiscence and reflection, a process universal for people of all ages from middle childhood [4]. However, reminiscence (looking back over our lives) is typically associated with old age [5], and reminiscence therapy has been shown to improve the mood of older people [6]. Reminiscence and story-telling are often triggered by mementos and collections in the home. Visual lifelog collections also have the potential to support story-telling. Byrne and Jones [7] investigated the narrative presentation of lifelog data through card-sorting tasks. Participants who were avid lifeloggers were asked to types of 'artifacts' that represented specific stories from their life. The study showed that participants used photographs (captured via a wearable camera) $50 \%$ of the time to support the narration of their experience. When questioned as to why this was the preferred method the participants reported that the lifelog images captured spontaneous moments in a life-like manner, which would not be typically taken with manual digital cameras.

These studies highlight the importance of reminiscing for people of all ages and suggest that sharing lifelogs could support reminiscence and story-telling. Some of the questions that we aim to address in this paper are:

- Does sharing motivate lifelog behavior?

- Does sharing influence browsing lifelog images?

- In what manner does sharing lifelog images influence browsing? 
These questions are answered in two phases. Phase 1 focuses on the first two questions, which we investigate through a long-term field study. Phase 2 involves a follow-up observation study and aims to understand how sharing influences browsing.

\section{RELATED WORK}

\section{A. Supporting Reminiscence and Reflection}

With the digitization of mementos such as photographs and videos, researchers have begun to realize the benefit of this media for reminiscence. Digital reminiscence systems have been developed to support conversation in people with dementia [8] using a combination of text, photographs, video and music from the past. Online tools such as YouTube have also been shown to support reminiscence in people with dementia, increasing well-being, mood and engagement in the activity compared to traditionally used methods [9]. Although older adults are less likely to use technology compared to younger individuals [10], Mulvenna and colleagues [11] found that older users had no significant issues when interacting with photographs on a device, compared to card-based photographs. One method of increasing older user acceptance of interactive reminiscence systems is to combine features from traditional objects, such as a music reel tape recorder, into an interface representation [12]; a technique referred to as skeuomorphic design. Similarly, digital artifacts such as FMRadio [13], the Living Memory Box [14], Family Archive [15] and Lovers’ Box [16] integrate both digital and physical components to support family reminiscence by creating an interactive experience.

Lifelog collections, such as emails, photographs, and context data (e.g. GPS) also have the potential to support reminiscence [17, 18, 19] and story-telling [7]. Taking the idea that the home is central to a memento collection, Kalnikate and Whittaker [19] developed a lifelog application, MemoryLane, which depicts different spaces in the home for digital collections through the interface design. Another system, Pensieve [17], supports reminiscence through memory triggers, sent by email containing content from personal social media services. The idea behind Pensieve was to support spontaneous reminiscence and reflection through digital memory prompts. Lifelogging devices also support reflection and story-telling within family groups. Lindley et al. [20] examined the use of a wearable camera with seven households over the course of a week. The device used automatically captured approximately 5,000 images per day, which is significantly more than a person would take manually. However, one of the findings that emerged from Lindley's study was that the family narratives when reviewing the lifelog images were similar to those expected when reviewing typical photographs. Following on from the initial study, Lindley et al. [21] explored how revisiting these images might foster reflection 18 months after capture. Reviewing the images allowed the participants to gain insight into their family life, routines and behaviors, prompting reflection on past activities and how life might be improved.

\section{B. Personal and Shared Family Archives}

Similar to shared reminiscence, artifacts and archives can represent both individual and shared experiences. For example, a person can share photographs from a school trip with their family, telling stories of events that happened and the people they met at the time. This same person could then share the photographs with a schoolmate who was also on the trip. This shared reminiscence will be very different from the first scenario, as the school mate will also remember the school trip, and can have an active input. Categorizing archives into personal or shared is not always straightforward. In this example, if the individual is a young child, then the mother in the family would probably consider the photographs to belong to the family collection. However, if the individual is an adult showing images from their childhood, then the collection might be considered personal. The meaning or value that we place on archives can evolve over time, or have different values for different people in a family. Sellen [22] identifies six different values that people place on home archives including: defining the self; honoring those we care about; connecting with the past; framing the family; fulfilling a duty; and purposeful forgetting.

Family archives are often governed by one person in the family, who manages and preserves collections for all individuals [23, 24]. Kirk and Sellen [25] found that it was typically the mother who made decisions about holding onto family artefacts, such as baby toys and clothes in households with young children. Lindley [24] carried out an interview study with eight adults over the age of 50 years who were currently involved in family archiving. She found that there are generally two types of family archives; those that are inherited and those that are created. In Lindley's study the participants were the "gatekeepers" of inherited family archives, taking responsibility to ensure that artifacts were not lost or damaged, were accessible and meaningful to younger generations using the information to make new discoveries about the family history. Inherited family archives were not considered to be owned by any one individual, and therefore family members were consulted before any alterations were made, such as adding onto the family tree. In contrast, records that were created from memory, such as memoirs, were considered to be personal, even when the purpose of creating the archive was to share with family. Creating personal archives allowed the participants to include information relevant to their own experiences, or from their perspective. In this study the participants considered personal items belonging to deceased parents, such as love letters, to be inherited by the family as a whole and that implied consent was given by their parents by the very act of saving the items when alive. Personal artifacts, such as letters or emails, are more likely to offer insight into an individual's experiences and personality, information which is cherished by bereaved family members [24, 26]. 
It has been established from the literature discussed that artifacts such as photographs enhance shared family reminiscence. The research has also shown that digital lifelogs can support story-telling, reflection and spontaneous reminiscence. The current study extends this understanding of lifelogging to investigate whether differences in lifelog behavior exist when people collect for sharing or personal purposes, and also whether reviewing lifelog images supports shared reminiscence between older and younger family members.

\section{PHASE 1: FIELD STUDY}

\section{A. Method}

The main goal of Phase 1 was to determine whether sharing was a motivating factor for lifelogging behavior of older adults compared to lifelogging for personal use. To do this we looked at both older and younger people's lifelogging behavior under sharing and non-sharing conditions. In this section we describe the tools used in the experiment, the participant profiles and the study procedure.

\section{1) Lifelogging tools}

Wearable camera: The lifelog data was recorded by the participants wearing a SenseCam (Fig. 1). The SenseCam is a wearable camera worn around the neck via a lanyard and sits around the chest area. Image capture is triggered by changes in the wearer's environment through embedded sensors monitoring light-intensity and light-color, temperature, movement and passive infrared. In addition, an internal timer automatically triggers image capture every 30 seconds. A fisheye lens maximizes the field of view so that images display almost everything within the wearer's field of vision.

Touch-screen browser: Each participant was given a touch-screen computer to allow them to upload the images from the wearable camera to custom browser software designed and developed within the research team [27]. To manage the high quantity of images that the camera captures the browser automatically segments the images into "events". An example event might be eating breakfast or driving a car. These events are represented by a key-frame image, an image taken from the middle of each event ${ }^{1}$. The browser interface was designed to accommodate older users with limited computer experience (Fig.1).

The browser allows users to upload images from a device connected to the computer, view a slideshow of images within an event, and navigate to different days using a calendar function. The main content area of the interface displays the key-frame images for a day, which a user can select to view more images within an event. The uploaded images are stored locally on the touch-screen computer, and once uploaded, automatically deleted from the wearable camera. The browser does not support online sharing, therefore 'sharing' for this study related to individuals viewing images from the same touch-screen system. The image count and frequency of user

\footnotetext{
${ }^{1}$ For more information see: http://sensecambrowser.codeplex.com/
}
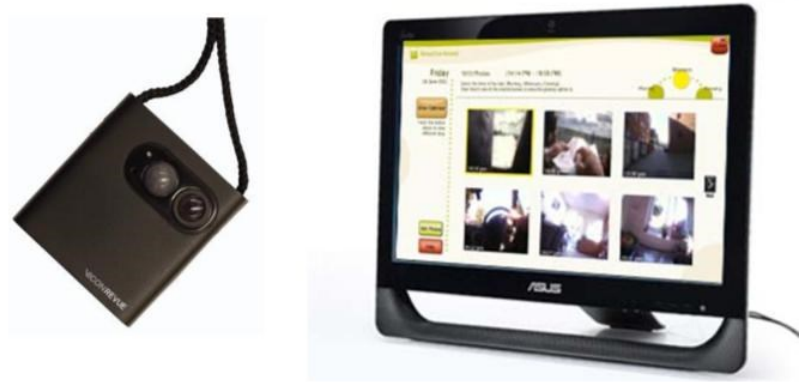

Figure.1 The SenseCam and browser on touch-screen PC [27].

interaction with the browser was automatically recorded using time and date-stamps. The user was also given documentation on how to use the browser should they need it.

Questionnaire: The participants were asked to complete a questionnaire at the end of each week. The questionnaire was identical for both sharing and non-sharing weeks, containing 15 items related to the participants' experience in viewing and sharing their lifelog images. This included how frequently they viewed or shared images, their enjoyment and factors that influenced their enjoyment.

\section{2) Participants}

The focus of the study was on family sharing therefore the participants were recruited as intergenerational family pairs. Ten family pairs participated in the study, 20 people in total with 10 older and 10 younger participants. Each older participant was a parent of one of the younger participants. Five of the younger adults were living in the family home with their parent. Overall the participants were familiar with technology, with all of the participants owning a mobile phone and having access to a computer.

\section{3) Procedure}

The participants were paired together with their family member so that there were 10 pairs of participants with each pair containing an older and younger participant. The study was conducted over a two week period during which they were asked to wear the camera and upload their images to the touch-screen browser. The purpose of the study was to examine whether sharing lifelog data had an effect on data capture. Therefore we divided the experiment into two separate weeks; one sharing and one non-sharing week. The order of these weeks was alternated for each group. For the "sharing week" the participants were asked to wear the camera for at least two days (this was to ensure that there was data to analyze); however they could wear the device intermittently and as frequently as they wanted. We wanted the participants to use the lifelog device according to their own preferences. When wearing the camera they were asked to undertake a common activity, such as going out for a coffee, with their paired participant. This was to ensure that there were images captured of a common event for both participants.

Participants were advised that they could share their lifelog data with other people, either their paired partner or anyone 
with whom they felt comfortable sharing. They could also choose not to share their images if they did not want to. At the end of the week the participants were asked to complete a questionnaire relating to their experience sharing or not sharing their photographs with others. The procedure for the "non-sharing week" was identical to the sharing week however the participants were advised to collect the visual lifelog data for their own personal viewing.

\section{B. Results}

The results of the Phase 1 study are presented detailing participant interaction with the wearable camera and browser and supported with the questionnaire findings.

\section{1) Interaction findings}

The image count and frequency of user interaction with the browser was automatically recorded using time and date stamps. This data was used to compare older and younger participant interaction for both sharing and non-sharing weeks in relation to the number of days the lifelog device was worn, the number of images captured, the time spent using the browser and the time spent logging. An increase in the time spent wearing the lifelogging camera results in an increase in image quantity. Therefore, motivation was measured by an increase, between the sharing and non-sharing weeks, in the recorded data for: days worn, time browsing, images captured and time logging. Table 1 displays the total values for each group over the two weeks. There was a slight decrease in the number of days that the camera was worn during the nonsharing week for both the older and younger group. The time spent browsing images also decreased during the non-sharing week, particularly for the younger adults. A total of 242,679 images were captured during this study with little variation between the two weeks. However there was variation in the number of images captured between the older and younger participants with older participants collecting a greater number than the younger group. As the embedded sensors within the camera can influence the frequency of image capture we also looked at the number of minutes that the participants wore the device. Again, the older group spent more time logging than the younger group and there was a decrease, albeit slight, in logging time for the non-sharing week.

\section{2) Questionnaire findings}

The questionnaire was used to collect information regarding participants' enjoyment of viewing and sharing their images. We were interested in determining what would motivate individuals to invest in this lifelogging effort, and also whether the participants would be more likely to lifelog for sharing purposes or for their own personal viewing. We were also interested in investigating what factors could contribute to the design of an application for intergenerational use. We report the findings in the following sections.

\section{a) Sharing lifelogs}

During the sharing week the participants could share their images with anyone that they felt comfortable sharing with, e.g. friends, family, and neighbors. In terms of who the
TABLE I. INTERACTION LOGS FOR PARTICIANTS WEARING SENSECAM AND USING BROSWER

\begin{tabular}{|l|r|r|r|r|}
\hline \multirow{2}{*}{ Total Recorded Data } & \multicolumn{2}{|c|}{ Sharing } & \multicolumn{2}{c|}{ Non-Sharing } \\
\cline { 2 - 5 } & \multicolumn{1}{|c|}{ Younger } & \multicolumn{1}{|c|}{ Older } & \multicolumn{1}{|c|}{ Younger } & \multicolumn{1}{c|}{ Older } \\
\hline Days Worn & 44 & 42 & 35 & 41 \\
\hline Time Browsing (mins) & 657 & 658 & 348 & 558 \\
\hline Images Captured & 53,365 & 74,110 & 49,584 & 65,620 \\
\hline Time Logging (mins) & 13,021 & 20,767 & 12,918 & 18,088 \\
\hline
\end{tabular}

participants were most likely to share their images with, we found that family members, including spouse, children and siblings were shared with most often. Furthermore, the type of images that were shared most often by both the younger and older participants featured family members. The participants rated their enjoyment for sharing the lifelog images as highly as their enjoyment for viewing the images alone (mean rating of 4 on 5-point-Likert scale). For example, one of the participants said that her husband asked to see her images and they looked through them together, laughing at the funny moments the wearable camera captured. The participants who were grandparents said that they particularly enjoyed sharing the images of their grandchildren. One particular participant shared his images with his spouse, his daughter and his grandchildren, which indicates lifelogging to be an intergenerational activity. The majority of the participants also said that they would like to keep their lifelogs to review in the future, to share with family or to pass on to their grandchildren. The motivations for holding onto these lifelogs echo the reasons given for collecting and saving physical mementos [22, 24]. These findings are very interesting to us as they show that sharing motivates lifelogging and suggests that lifelogs could support shared family reminiscence.

Not all of the participants shared their images during the sharing week. From the questionnaire responses it was clear that some of the participants considered their images to be uninteresting. Images taken from activities such as driving, working at a computer or watching the television were considered to be particularly "boring”. If the study had been carried out over a longer period it is probable that certain activities would stand out as being interesting and therefore worth sharing. A potential benefit of capturing lifestyle patterns is the possibility to reflect on the time spent on certain activities, perhaps influencing positive lifestyle change. Another problem that a few of the participants faced during the sharing week was finding a person who was interested in looking at the images. For example, one of the participants told us that there were images taken of their friend that they wanted to share, however they were not in contact with the person again during the sharing week so they were unable to show them. Again, in a real-world situation the participant could share these images the next time they saw their friend, which might be weeks or months after the event. 
Other issues that influenced whether people shared their images were more technical and included the poor quality of the images and re-finding images to share. Images recorded with the wearable camera are affected by motion blur and low level natural light and therefore when hundreds of images are taken during an activity that causes this problem it can become frustrating to a user browsing through the collection. Ideally, very low quality or dark images would automatically be hidden or deleted depending on the user's preferences. Similarly, the large number of images means that when a user returns to their collection to find a particular image it can be very difficult. Although the image browser automatically groups visual lifelog collections by date, time and event, the user is still required to remember these details. Naaman et al. [28] recommend the use of context-based features, such as location, for photo management. The version of the SenseCam used in this study did not capture location based information, however other image capture devices, such as mobile phones and more recently developed wearable cameras (e.g. Memoto, Autographer), already have GPS integration.

\section{b) Age differences}

Some age-related differences emerged from the study that could impact the future design of an intergenerational lifelog application. One of the findings from the results was that the older participants spent more time lifelogging than the younger participants. The reasons behind this became obvious when we looked at the reports on why they did not wear the camera at certain times. For example, most of the older participants were retired so they could choose to wear the device whenever they liked. In contrast, many of the younger participants said that they could not wear the device in the workplace or at meetings. Furthermore, remembering to put on the camera after working hours proved to be a challenge, for both older and younger participants who were working.

Another age-related difference that we found from the results was how the younger participants included friends more in their lifelog experiences. The younger participants reported friends featuring in their lifelog images twice as often as the older group. The younger group also shared their images with friends whereas the older group only shared with family. The younger participants were also more experienced with technology. Although the number of older Internet users is fast growing, younger people are still the largest user group [29]. This is also true for social networking sites such as Facebook and Twitter set up for sharing information and photographs, so it is likely that our younger participants would have more experience sharing through social networking sites than the older participants. The younger participants also said that they would have shared their lifelog images more if they had been able to share them through the Internet.

\section{c) Lifelogging experience}

Using an automatic camera was a new experience for the participants in this study. The experiences support those found in the study by Lindley et al. of SenseCam use [20]. In Lindley's work the experiences of seven households, five of whom had young children, centered on routine, playfulness and sense of family. The participants in the current study also observed the repetitiveness of their activities from day-to-day and the time that they spend on particular activities that they don't enjoy. Unusual events were highlighted in the participant's mind because they occurred outside of this routine, such as viewing a house for sale and witnessing a car crash. In the current study, playfulness was not as evident, however the participants did comment on how they found it fun wearing the lifelog device and looking through their images. Some of the participants also said that they placed the camera in different areas of the home to capture their household activities from a different perspective. Sense of family was again echoed by our study in that the participants' images mostly featured family, they showed a preference for sharing with family members and they showed an interest in keeping their images to pass down to family members.

In this study we looked at whether older and younger family members would be more motivated to lifelog for sharing or personal browsing purposes. It was found that although the participants wore the automated camera more frequently during the sharing week, certain factors inhibited sharing between participants, such as lack of access to the family member's images. Another issue raised was that the participants did not find their images worth sharing because the activities were so recent, and that they would have more interest viewing and sharing their images at a date in the future when the events were less clear in their mind. Therefore to further investigate family sharing Phase 2 was set up.

\section{PHASE 2: FOLLOW-UP OBSERVATION}

Phase 2 was a follow-up observation study of participant families browsing through and sharing their visual lifelog collection six months after data capture. The purpose of this observation study was to explore whether reminiscence and story-telling is supported by lifelog sharing. This study was influenced by the research of Lindley et al. [21], which involved observing young householders reflecting on their lifelog images 18 months after data capture

\section{A. Method}

\section{1) Participants}

Eight of the participants who had taken part in the field study were available to participate in the follow-up study. For ease of discussion these families will be referred to as Fam1 to Fam4. A short description of these families is outlined below:

- $\quad$ Fam1 is comprised of a father (Fam1-f) aged 66 years old and his daughter (Fam1-d) aged 36. The father lives with his wife and minds his grandchildren during the week when his daughter is working in a school. The daughter is married and living with her husband with two children.

- $\quad$ Fam2 is comprised of a father (Fam2-f) aged 64 and his daughter (Fam2-d) aged 29. The father is widowed, living with his son and retired. The 
daughter is living with her partner and working in a web-design company.

- $\quad$ Fam3 is comprised of a mother (Fam3-m) aged 62 and her daughter (Fam3-d) aged 29. The mother is divorced, living with her second daughter and working part-time in a hospital. The daughter is living with friends and working in an insurance company.

- $\quad$ Fam4 is comprised of a father (Fam4-f) aged 58 and his son (Fam4-s) aged 30. The father lives with his wife and is semi-retired. The son is married, living with his wife and working in a university.

\section{2) Procedure}

An observation method was used in the follow-up study to explore participants reviewing their lifelog images together at least 6 months after capture (the interval varied by 2-3 weeks). Observations were conducted in the home of the older participant of each family. The participants were given the choice of setting so that they would feel comfortable reviewing the images and to encourage natural behavior. Before the observation study began the participants were reminded how to use the visual lifelog browser used in the Phase 1 field study. At this stage the participants were offered the opportunity to browse through their images privately to ensure they were happy to share their images. None of the participants chose to partake in private browsing.

The participants were then asked to browse through the images together. They were told that they could choose who would go first. They were advised that they did not have to look through all of the images if they did not want to. The participants were also advised that either family member could control the interactions with the browser. The participants were reminded that the researcher was only there to passively observe and that they should behave as naturally as possible.

Field notes were taken to record the participants interactions. An audio recording was also taken to support the field notes. The observations focused on the dialogue between the participants when browsing the images. We analyzed the field notes using inductive thematic analysis. The data was coded, memos written, and patterns identified. On the basis of this we derived the findings.

\section{B. Observation Findings}

The purpose of the observation study was to determine whether browsing and sharing lifelog images supported shared family reminiscence. Reminiscence was indeed observed throughout this session. However, in addition to reminiscence narration, story-telling, reflection, and interaction between the family members was observed. We now discuss these observations in more detail.

\section{1) Narration, reminiscence and story-telling}

Narration is commentary delivered to accompany a visual display, such as a video, play or in the current context an image slideshow. Narration was the method of interaction that dominated the observed browsing sessions. This was particularly evident at the beginning of an event or set of images being looked at. The process of narration was usually a step-by-step dialogue of observed actions, such as: "there I am washing up.... back in the garage...feeding the dogs" (Fam1-f). It was observed that the person who interacted with the browser led this narration, regardless of whether they owned the images or not. If the narrator was not familiar with the other person's location or actions within the lifelog, they would stop to ask questions, "you're in the garage, what were you getting?” (Fam1-d). These narrations provided the basis for family interaction and it was from this that reminiscence, storytelling and reflection intermittently occurred.

Reminiscence was observed where the lifelog images triggered memories of a past event. This was particularly evident where the participant's circumstances had changed since the images were first captured. For example, both participants in Fam3 had captured images of the daughter preparing to move out of the family home. The whole family was involved in helping to pack up boxes, move them out and set her up in her new home. Browsing through these images encouraged the parent and child to reminisce about this time, the struggles and the fun they had. Shared reminiscence most often occurred when family activities were captured. These were often amusing memories, for example, when Fam1 were browsing through images of a family dinner in a restaurant, the daughter remembered that her child had taken the mussel shells from a pasta dish home to put in their garden, where she liked to collect shells, branches and flowers.

Not all of the memories that were evoked from the images were positive, however. On one occasion a participant said that they did not want to be reminded of a specific experience he had. The image reminded him of how frustrated and annoyed he was at the time. This indicates the importance of providing the user with control over their lifelogs, so that images can be deleted if the memory is upsetting.

Story-telling occurred when the participants came upon images of an event that they particularly liked, or an event that was important to them at the time. The images were used to support story-telling. For example, at the time of data capture one of the older participants, Fam2-f, was getting his house renovated and had visited different show houses to get ideas. He had given his daughter a verbal account of these after his visits, however when they were browsing through his lifelog they came across images of this event and he became animated, saying to his daughter, "slow it down, this is the house I wanted you to see". This led to an elaborate story about his experience in the house, the design features, the people who were there, and how the house contrasted to their now renovated home.

It was common for a set of images to trigger stories about the event that was taking place. Story-telling was used to provide an explanation of why the participant was doing specific activities, filling in the details that the wearable camera did not capture, such as the conversations that were occurring, or the mood in the room. In the browsing session 
with Fam4, the son was sharing his images with his father. He noticed that in the images he was cooking for an unusually long time. After looking at the date he remembered that it was Valentine's Day, and that he was preparing a three course meal for his wife. He expanded by telling his father what he was cooking, how he found the recipes and where his wife was at the time he was cooking. This event had occurred six months previously and even though it was a significant memory for the younger participant (from the observed enthusiasm in his story-telling); he had not shared this story with his father before the browsing session.

The observations show how narration, reminiscence and story-telling can enhance lifelog browsing and in turn, how lifelogs support these processes.

\section{2) Reflecting on activities and behaviours}

As mentioned in Section II, Lindley et al. [21] previously explored householder's reflections on visual lifelog data 18 months after data capture. In comparison to the current study, Lindley's participants were either young couples or families with young children. However, the results obtained from Lindley's research mirrors the observations found in the current study, particularly in relation to reflections on routine, reflections on behaviors, and the changing value of the lifelog images over time.

When the participants reviewed their images it was the activities of the wearer and those around them that were of most interest. Some images did not immediately trigger a memory of the event. When reviewing these events, the participants were determined to figure out what they were doing, where and why, using clues in the images and the time it was taken to help them reach a conclusion. Talking to the participants after the browsing session, it was found that they preferred reviewing the lifelogs images with their family because they enjoyed trying to figure out these forgotten activities. The daughter in Fam2 for example, could not understand why everyone in her images was eating cake during the day. After looking at the date on the browser she realized that it was her brother's birthday; "We're eating cake...Oh its John's birthday, November. No wonder he's being sociable".

Time was a significant component for reflecting on activities. This included references to the time that the images were recorded (time of day, month etc.) and the time taken to complete tasks. It was observed that the participants continuously made a note of the time, especially when trying to determine what activities they might be doing in the images; “It's seven o'clock, I must be on my way to the club". The participants also made use of time to report how long the camera wearer or other people in the images had taken to complete their tasks. There were numerous examples of this. The daughter in Fam1observed how long it took for her father to pack everything into his van when he was going camping, and Fam4-s used the images to show how long he was left waiting on a family outing while everyone went shopping. Similarly, the daughter in Fam3 came across images of her brother putting together a bedroom cabinet for her. The mother and daughter joked that he spent ten hours putting it together. However, when they were looking through the images they realized that he only took two hours and the rest of the family had stood around watching him. They reflected that maybe they should not have teased him so much about it.

Although the participants' lifelogs were made up of irregular events, such as renovating a house, moving out of the family home, dinners and nights out with friends and family, the participants were surprised at the predictability and sameness of their lifestyle. They voiced their observations concerning patterns and behaviors; "Get up, have breakfast, go out. Every day is the same" (Fam1-f). Routine was even observed in the types of food they ate; "I'm mixing up tuna and pitta again. We went through a phase of eating that" (Fam1-d). The younger participants were surprised that their own lifelogs depicted routine and sameness more so than their parents'. They had not expected their parent to be more social and active than they were; "my day isn't as exciting as yours" (Fam3-d). After reviewing the images, the participants observed activities that they believed they spent too much time doing, such as household chores, watching TV and sitting at a computer. One participant reflected that although she spends many hours doing housework, this was not likely to change; "I do a lot of clothes and mundane jobs. That's not going to change though" (Fam1-d).

Personal behaviors and habits were also observed and commented upon throughout the browsing session. This was in relation to the participant's own behaviors, those of their parent/child, or other people captured in the images. The images either reaffirmed the participants' beliefs or produced previously unknown behaviors. For the most part these observations were commented on in jest; "look at you, you do a lot of hand movements when you're on the phone don't you?" (Fam2-d). Other behaviors that the participants pointed out were texting in the car, playing with a pen in meetings, or sitting tensely. For example, the father and daughter in Fam2 were browsing through the father's images, which showed him watching TV when the daughter commented on them:

Fam2-d - That's a very tense way to sit. You shouldn't be sitting like that.

Fam2-f - Oh, it's a match.

Fam2-d - Oh, rugby.

Fam2-f - That's what it was, it was a rugby match.

Fam2-d - All you can see is the hands like this (shows hands clenched together) in front of the SenseCam.

In this scenario, it was only after the participants browsed through the event that they saw the rugby match on the television and realized that this was why the father was sitting so tensely. 


\section{3) Family interactions}

It was observed that narration and reflection on activities came about with or without family interaction. That is, one person could give an account of their activities without any participation from their parent/child. Reminiscence and storytelling in contrast transpired through family interaction, enhanced by a teasing rapport between the family members. In other words, the participants were encouraged to share their memories and experiences by their family member, through questions or teasing them about activities or behaviors. Having a shared interest also encouraged this interaction. This could have been having images of the same event, seeing themselves in the other person's images, or seeing images of family or shared acquaintances.

After the browsing sessions we briefly discussed with the participants their opinion on sharing their images together or whether they would have preferred to share them remotely or not at all. Six of the eight participants said that they considerably favored browsing through the images together; "together definitely, you can ask questions and have a bit of fun" (Fam1-f). For these participants teasing and poking fun was prominent throughout their interactions; "your hand looks old like mine, old and wrinkly" (Fam3-m). In contrast, the remaining two participants said that they would feel more comfortable sharing their images remotely, i.e. not in person, so that their family could look through them in their own time and of their own accord. This finding suggests that a family browser should support both types of sharing; in person and remote sharing. There was also some variation in responses for sharing lifelogs. Most of the participants said they would prefer to choose events to share rather than allowing their family to have open access. Even the participants who said they would not mind their immediate family having access were wary against extended family or in-laws having or asking for access. Finally, although the participants said they would love to have a lifelog from their deceased parents or grandparents, they said that they would only want to leave behind lifelogs that they had chosen to share when living.

\section{SUPPORTING SHARED FAMILY REMINISCENCE: DESIGN CONSIDERATIONS}

The findings from these studies show that sharing is a motivation for people to lifelog. However people also enjoy viewing their own images, seeing the things they did and the people they were with. Therefore, we believe that a system that supports shared family reminiscence must display the user's own personal collection, enhanced by the family's lifelog collections. Incorporating the findings from this study we have identified three approaches that we believe could achieve this. These are: 1) highlighting events and activities; 2) combining lifelogs; and 3) family lifelog. Fig. 2 displays how these methods could be used in an interface display.

\section{A. Highlighting Events and Activities}

The activities in which the participants in the field study were involved influenced their interest in viewing and sharing lifelogs. Events that involved other people or unusual activities were of interest, whereas other inactive events such as sitting in front of a computer or driving were considered boring. However, some activities that were initially described as boring were later discovered to be interesting and revealed to the participants how much time they spend on various activities (e.g. cleaning). Highlighting events and activities would allow users to quickly find interesting images, or share images that they think would interest others. This could be automatically achieved in three ways (see left image in Fig. 2);

\section{1) Highlighting important events}

One of the problems reported by the participants who used the lifelog browser throughout the field study was that they had difficulty refinding images to share, amongst the large quantity of images captured. An easy solution for this is to provide users with the option to highlight images or events that they want to retrieve again, such as marking an event as 'favorite'. This would duplicate the favored images from the main data set, copying it (or a symbolic link to it) into a separate folder to which the user has direct access. In a large data set collected over months or years, images/events marked
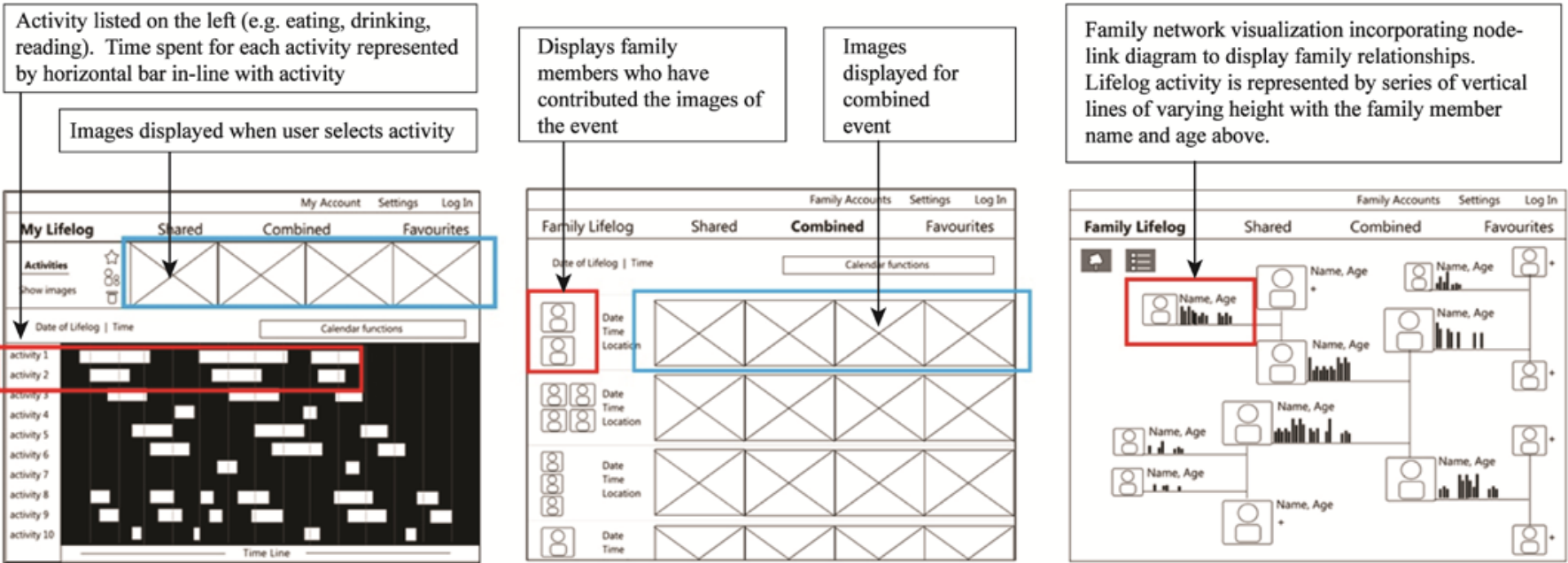

Figure 2. Proposed visualizations for (1) highlighting events and activities, (2) combining lifelogs, and (3) family lifelog 
as 'favorite' may be difficult to retrieve. Therefore, within this data set, search and retrieval techniques should support users to quickly and easily find desired images or events.

\section{2) Recognize event activity}

Lifelogs provide users with a detailed representation of their everyday activities. Doherty et al. developed a technique for automatically eliciting these activities, or lifestyle traits, from visual lifelogs [30]. This is achieved through the extraction of image features, which are analyzed for pattern recognition, and then used to classify lifestyle traits, such as reading, walking, eating, or being with other people. This allows users to easily find events that may be of interest to them, such as meeting friends or having dinner in a restaurant. Although this approach is under development, for applications in the domain of health and lifestyle, implementation into a lifelog browser to be deployed with nonexpert users is not yet possible due to the current performance of such techniques.

\section{3) Search based}

Additionally, Doherty et al. recommend providing search functionality for 'who, what, when and where' to enhance the lifelog browsing experience [31]. Image retrieval through search can be text-based, through manual tagging or using the information obtained from image features. To improve search functionality with wearable cameras, Qiu and colleagues [3] have explored the use of accelerometer data to provide the context of a user's activities. Additional functions, such as GPS and Bluetooth for example, can significantly enhance search performance; however these sensors are not yet incorporated within the SenseCam device used in the current study, although early stage research and development effort is looking at alternatives that do incorporate these sensors [3].

\section{B. Combining Lifelogs}

We found from the results of the studies that given the opportunity, the participants enjoyed viewing their younger/older family member's lifelog images. Viewing these images allowed them to see what the other person did throughout a normal day. It also allowed them to see events from a perspective other than their own. Images that the participants also enjoyed viewing were those featuring family members. To support these preferences we recommend that a family lifelog browser allow users to combine their lifelogs (see middle image of Fig. 2). For example, if a user has images of an event, say their birthday party, and other family members shared their images of the same event, then the user could combine these images enhancing their own image set. Automatically identifying similar events from family members' lifelogs could be implemented using Doherty et al.'s event association approach [31]. Automatic event association is achieved by firstly identifying an image's traits, linking the images by time in a first step and then by visual features, typically color, edge and texture properties, in addition to a number of other traits. These representative vectors are then compared against each other to determine the similarity between events. Doherty and Smeaton [32] showed how lifelogs can be augmented by combining external sources from the Web and integrating them into one's own lifelog.
The same principle could be applied for combining family events, albeit perhaps requiring more sophisticated object/scene/person matching. This opens up a whole new research question which future studies should clearly investigate further.

\section{Family Lifelog}

To support family-based lifelogging we not only have to think about the user and their lifelog but also the family as a whole. Shared reminiscence helps to create bonds between family members and maintain memories of past loved ones [4]. The idea behind the family lifelog is to include, through a family tree like structure, the lifelogs of all family members, past and present (see right image of Fig. 2). A user would only see the lifelog images that a family member has shared. We believe it is necessary to allow users to have the option to share with the whole family network, specific family groups or individual people. Similar to the user's personal lifelog, the family lifelog would integrate event importance and event association methods to allow the user to identify events of interest in their family lifelog. A user could see activities in which their grandparents had participated and perhaps share these images with their own children. Considerations would also have to take into account user preferences for lifelog remains, namely who would have access to the lifelogs and the level of privacy expected.

\section{Limitations of Research}

Similar to previous studies [13, 15, 21] our research highlighted the potential of digital family archives to bring family members together, sharing and reflecting on the recorded data. However, family relationships are constantly changing, through the birth of children, death, or divorce for example. Throughout the study period our participants did not experience any significant changes in their relationships, and were happy to share their lifelog images with each other. It is expected that long-term lifelogging would raise many more issues, from relatively minor issues such as accidently sharing images, to serious issues, such as marital problems, using lifelog images to monitor a spouse's activities. Odom et al. [33] claims that characterizing and understanding family life is complex, however families work at adapting to these structure shifts and technology is becoming a common part of this process. Odom suggests that more research is needed to fully understand the role of digital possessions following family loss or marital breakdown. This would also be relevant to lifelog contents shared within families.

Another issue that should also be considered for personal lifelog information within a family application is 'lifelog remains'. In other words, what happens to the data when the user is deceased; is it automatically deleted, do family members inherit it, or should the user have a digital will of some sort? Previous research has shown that in the absence of explicit instructions, family members of deceased individuals use their own discretion over what they think their family member would consider private within their digital collections [34]. Privacy in digital collections is more ambiguous 
compared to physical items such as a diary or personal letters. This topic could be explored in more detail with a specific emphasis on visual lifelogs.

\section{CONCLUSION}

In conclusion, we have determined through the field experiment and observation study that sharing is a motivating factor for lifelogging and that family-based sharing supports family interaction through reminiscence, narration, storytelling and reflection. We use these findings to inform the design of an intergenerational family lifelog browser. The designs proposed contribute to an understanding of how extremely large data sets should be presented to users to allow them to browse, find and share interesting images. In a lifetime, a person would accumulate millions of images, therefore the way in which this data is presented is very important. The interface design and visualization techniques presented here, which are supported by the empirical research studies, offer a solution to the problems associated with the display of large lifelog data sets.

\section{ACKNOWLEDGMENT}

The funding for this research has been provided by the IRCSET. This work is also supported by Science Foundation Ireland under grant 07/CE/I1147.

\section{REFERENCES}

[1] G. Lanzieri, "The greying of the baby boomers: A century-long view of ageing in European populations”, retrieved March, 2012 from http://epp.eurostat.ec.europa.eu/portal/page/portal/product_details/public ation?p_product_code=KS-SF-11-023, 2011.

[2] D. De Jager et al., "A low-power, distributed, pervasive healthcare system for supporting memory”, Proc. MobileHealth 2011, art. 5.

[3] Z. Qiu, C. Gurrin, A.R. Doherty, and A.F. Smeaton, "A real-time life experience logging tool”. Proc. 18th MMM Conference, 2012.

[4] J.D. Webster, and M.E. McCall, "Reminiscence functions across adulthood. A replication and extension”, J. of Adult Dev., vol. 6, 1999, 73-85.

[5] R.J. Havighurst, and R. Glasser, “An exploratory study of reminiscence”, J. Gerontology, vol. 27, 1972, 245-253.

[6] B. Woods, A. Spector, C. Jones, M. Orrell, and S. Davies, "Reminiscence therapy for dementia", Cochrane Database Syst. Rev., vol 18, CD001120, 2005.

[7] D. Byrne, and G.J.F. Jones, "Exploring narrative presentation for large multimodal lifelog collections through card sorting”, Proc. Interactive Storytelling, 2009, p. 92-97.

[8] A.J. Astell et al, "Using a touch screen computer to support relationships between people with dementia and caregivers”, Interact. Comput., vol 22, 2010, pp. 267-275.

[9] J.O. O’Rourke, F. Tobin, S. O’Callaghan, R. Sowman, and D.R. Collins, "“YouTube': A useful tool for reminiscence therapy?" Age and Ageing, vol. 40, 2011, pp.742-758.

[10] S.J. Czaja et al., "Factors predicting the use of technology: Findings from the Center for Research and Education on Aging and Technology Enhancement (CREATE). Psychol. Aging 21 (2006) 333-352.

[11] M.D. Mulvenna et al., "Evaluation of card-based versus device-based reminiscing using photographic images”, J. CyberTherapy Rehabil., vol. 4, 2011, pp. 57-66.

[12] A.J. Astell, N. Alm, G. Gowans, M. Ellis, R. Dye, and P. Vaughan, "Involving older people with dementia and their carers in designing computer based support systems: Some methodological considerations”, Univ. Access Inf. Soc., vol. 8, 2009, pp. 49-58.

[13] D. Petrelli, N. Villar, V. Kalnikaite, L. Dib, and S. Whittaker, "FM radio: Family interplay with sonic mementos”, Proc. CHI 2010, p. 23712380.

[14] M.M. Stevens, G.D. Abowd, K.N. Truong, and F. Vollmer, "Getting into the living memory box: Family archives \& holistic design”, Pers. Ubiquit. Comput., vol. 7, 2003, pp. 210-216.

[15] D. Kirk, S. Izadi, A. Sellen, S. Taylor, R. Banks, and O. Hilliges, Opening up the family archive, Proc. of CSCW 2010, p. 261-270.

[16] A. Thieme, J. Wallace, J. Thomas, K.L. Chen, N. Kramer, and P. Olivier, “Lovers' box: Designing for reflection within romantic relationships”, Int. J. Hum-Comput. Stud., vol. 69, 2011, pp. 283-297.

[17] D. Cosley, V.S. Sosik, J. Schiltz, S.T. Peesapati, and S. Lee, "Experiences with designing tools for everyday reminiscing", HumComput. Interact. 27 (2012) 175-198.

[18] B. Kikhia, J. Hallberg, J.E. Bengtsson, S. Sävenstedt, and K. Synnes, "Building digital life stories for memory support", Inform. Syst. J., vol. 1, 2010, pp. 161-176.

[19] V. Kalnikaite, and S. Whittaker, “A saunter down memory lane: Digital reflection on personal mementos”, Int. J. of Hum-Comput. Stud., vol. 69, 2011, pp. 298-310.

[20] S.E. Lindley, D. Randall, W. Sharrock, M. Glancy, N. Smyth, and R. Harper, "Narrative, memory and practice: Tensions and choices in the use of a digital artefact”, Proc. BCS-HCI 2009, p.1-9.

[21] S.E. Lindley, M. Glancy, R. Harper, D. Randall, and N. Smyth, "Oh and how things just don't change, the more things stay the same: Reflections on SenseCam images 18 months after capture”, Int. J. Hum-Comput. Stud., vol. 69, 2011, pp. 311-323.

[22] A. Sellen, Family archiving in the digital age, in: R.H.R. Harper (Ed.), The Connected Home: The Future of Domestic Life, Springer, London, 2011, pp. 203-236.

[23] A. Durrant, D. Frohlich, A. Sellen, and E. Lyons, "Home curation versus teenage photography: Photo displays in the family home", Int. J. HumComput. Stud., vol. 67, 2009, pp. 1005-1023.

[24] S.E. Lindley, "Before I forget: From personal memory to family history”, Hum-Comput. Interact., vol. 27, 2012, pp. 13-36.

[25] D. Kirk, and A. Sellen, "On human remains: Value and practice in the home archiving of cherished objects”, ACM Trans. Comp-Hum. Interact., vol. 17, 2010, p. 10.

[26] M. Massimi, and R. Baecker, "A death in the family: Opportunities for designing technologies for the bereaved”. Proc. CHI 2010, p. 18211830.

[27] N. Caprani, A.R. Doherty, H. Lee, A.F. Smeaton, N.E. O’Connor, and C. Gurrin, "Designing a touch-screen SenseCam browser to support an ageing population”, Proc. CHI '10 Ext. Abstracts, pp. 4291-4296.

[28] M. Naaman, S. Harada, Q.Y. Wang, H. Garcia-Molina, and A. Paepcke, "Context data in geo-referenced digital photo collections," Proc. ACM Multimedia 2004, p. 196-203.

[29] K. Zickuhr, “Generations 2010: Pew Internet \& American Life Project”, retrieved March, 2012 from http://pewinternet.org/Reports/2010/Generations-2010.aspx 2010.

[30] A.R. Doherty, et al., "Passively recognising human activities through lifelogging”, Computers in Human Behavior, vol 27, 2011, pp. 19481958.

[31] A.R. Doherty et al., "Experiences of aiding autobiographical memory using the SenseCam”, Hum-Comput. Interact., vol. 27, 2012, pp. 151174.

[32] A.R. Doherty and A.F. Smeaton, “Automatically augmenting lifelog events using pervasively generated content from millions of people”, Sensors, vol. 10, 2010, pp. 1423-1446.

[33] W. Odom et al., Absence and family life: understanding and supporting dynamic adaptation to change, in: R.H.R. Harper (Ed.) The Connected Home: The Future of Domestic Life, Springer, London, 2011, 237-266.

[34] M. Massimi and R. Baecker, “A death in the family: Opportunities for designing technologies for the bereaved”, Proc. CHI 2010, p. 18211830. 